\title{
Digital implant dentistry A to Z
}

\author{
Heekyung Lee $\mathrm{e}^{1,2}$ \\ 'Leeheekyung Dental Art Clinic, Seongnam, ${ }^{2}$ Vice-president of Korea Academy of Implant Dentistry, Seoul, Korea
}

In 1965, the study for osseointegration of titanium implants, published by Dr. Branemark, marked an innovative paradigm shift in terms of dental rehabilitation for edentulous. Currently, dental implant treatment become a common practice in our clinical field. The clinical application of dental implants has ranged from the maintenance and support of the denture, and the functional rehabilitation of partial edentulous to the aesthetic recovery. As the advancement of digital technology, a digital workflow has been established from diagnosis and planning to prosthesis of the implant rehabilitation. Thus, the era of 'Digital Implant Dentistry' has opened wide.
With the theme of 'Digital Implant Dentistry A to Z' at the 2020 Fall Conference of the Korean Academy of Implant Dentistry (KAID), the entire workflow of 'Digital Implant Dentistry' from diagnosis and surgical planning to guide production, surgery and prosthesis, was clearly summarized. Furthermore, the professors, who presented at the Fall Conference of KAID under the slogan of 'Back to the basic, challenge to the future', academically organized the research papers in JDIR. For the readers of JDIR, there will have great opportunity to understand and gauge the advance of digital implant dentistry, in the cutting edge. 